\title{
Perturbation Analysis of Spindle Speed Variation in Machine Tool Chatter
}

\author{
M. PAKDEMIRLI \\ A. G. ULSOY \\ Department of Mechanical Engineering and Applied Mechanics, University of Michigan, 2266 \\ G. G. Brown Building, Ann Arbor, MI 48109-2125, USA
}

(Received 29 July 1996, accepted 13 December 1996)

\begin{abstract}
Spindle speed variation has been shown to be an effective method for chatter control. In this paper, a single-degree-of-freedom regenerative type chatter equation is treated using perturbation methods. Rather than using the time coordinate, the angle of revolution is taken as the independent coordinate for maintaining a constant delay in the equations. The spindle speed is taken to be harmonically varying about a constant mean speed. Approximate analytical solutions are sought using the method of strained parameters, a perturbation technique. The amplitude of speed fluctuations $(\varepsilon)$ is assumed to be small, and solutions are constructed using this parameter as the perturbation parameter. The stability lobes for constant spindle speeds are calculated exactly. By using the approximate perturbation analysis, the gain in stability is calculated for variable spindle speeds. The analysis is valid for (E) values up to 0.02 (i.e., $2 \%$ of the constant mean speed). Solutions are verified using numerical simulations of the original equation.
\end{abstract}

Key Words: Perturbation methods, machine tools, chatter, speed variation

\section{INTRODUCTION}

A fundamental problem in machine tool dynamics is the elimination of chatter vibrations. Chatter reduces the tool life, causes poor surface finish, and leads to alignment problems and damaged bearings. Extensive research has been done on understanding the mechanisms behind chatter and on the elimination of unwanted vibrations. The fundamentals of chatter are outlined in Arnold (1946), Tobias (1965), and Welbourn and Smith (1970). Three basic mechanisms are known to be effective in causing chatter: (1) mode coupling, (2) velocity dependent effect, and (3) regenerative effect. Among these three, the regenerative effect is the most influential. When a cutter removes chips from the workpiece, a wavy surface is left behind. In the next turn, another wavy surface, out of phase with the previous one, causes variations in the depth of cut, which then alters the cutting forces. These alterations in the cutting forces due to tool and workpiece interactions lead to self-excited vibrations. When the amplitudes of these vibrations become large, chatter occurs.

One of the effective ways of reducing chatter is to vary the spindle speed continuously. This method was first proposed by Stoferle and Grab (1972). Inamura and Sata 
(1974) then used function space theory to understand the effectiveness of this method. Due to some oversimplifying assumptions, their method predicted gains in stability much higher than those numerically and experimentally observed. Sexton, Milne, and Stone (1977) repeated their analysis, corrected the unjustified assumptions, and found moderate improvements. However, the results they found are qualitatively different from their analog computer simulations (Sexton and Stone, 1978). Although theoretically they determined stability lobes, they could not find definite lobes in the case of analog computer simulations. Takemura, Kitamura, and Hoshi (1974), Inamura and Sata (1975), and Hoshi, et al. (1977) experimentally investigated the suppression of chatter by spindle speed variation. Sexton and Stone (1980) predicted the transient vibration behavior, which may be harmful even though the system reaches a final stable solution, through experiments and analog computer simulations. Lin, DeVor, and Kapoor (1990) investigated the spindle drive characteristics and showed that sinusoidal variations can be tracked more precisely than some other periodic waves in practice. Their experimental and numerical simulations proved that vibrations can be suppressed substantially by continuously varying the spindle speed. They searched for optimum values of fluctuation amplitudes and frequencies of the spindle speed. Tsao, McCarthy, and Kapoor (1993) considered a single-degree-of-freedom regenerative chatter equation in revolution angle coordinates rather than using time as the independent variable. This formulation transforms the timevarying delay equations into a system with fixed delay. A finite difference scheme is used in analyzing the stability of the system. Stability lobes are calculated numerically for variable speeds.

One of the inadequacies of the linear theory is that the unstable vibrations grow indefinitely, which is a contradiction of what is observed experimentally. Two mechanisms to overcome the discrepancy have been proposed: one is the multiple regenerative effect and the other is the nonlinearities of both structural and regenerative type. Kondo, Kawano, and Sato (1981) investigated the multiple regenerative effect. Lin and Weng (1990) developed a nonlinear model and solved this model using the method of multiple scales, a perturbation technique. Nayfeh, Chin, and Pratt (1996) used another nonlinear model first proposed by Hanna and Tobias (1974). The model includes the quadratic and cubic stiffness terms of the machine tool as well as linear, quadratic, and cubic regenerative terms. Using the method of multiple scales, the harmonic balance method, and direct numerical integration, they predicted limit-cycle, quasi-periodic, and chaotic behavior of this model.

Perturbation methods are very effective in analyzing vibration phenomena. Because they yield approximate analytical solutions, a better understanding of the problem can be achieved when combined with numerical techniques. In this work, for the first time, we apply perturbation techniques to the variable spindle speed chatter problem. The nonlinear analyses by Nayfeh, Chin, and Pratt (1996) and Lin and Weng (1990) do not address the variable spindle speed case.

In this paper, we apply perturbation methods to obtain approximate solutions to the chatter problem with spindle speed variations. Following Tsao, McCarthy, and Kapoor (1993), we consider a single-degree-of-freedom linear chatter equation in revolution angle coordinates, since the fixed delay equations are more appropriate for a perturbation analysis. This is the basic model (i.e., linear, single degree of freedom, single regenerative 
effect) widely used for the analyis of chatter vibrations in turning, and we employ it here as well. The method of strained parameters is used as our perturbation technique. The increases in the stability regions compared to the constant speed problem are calculated for variable spindle speeds. The perturbation parameter is selected as the amplitude of the speed fluctuations. We compare our results to those presented by previous researchers. We found that the proposed perturbation analysis is only valid for very small amplitudes of the speed fluctuations. Resonant frequencies to be avoided are determined. Results are verified using numerical integrations of the original equations.

\section{MODELING AND ANALYSIS}

In this section, we treat the one-dimensional linear regenerative type chatter equation expressed in angle coordinates. The spindle speed is assumed to vary harmonically about a constant mean speed. Approximate analytical solutions are presented using the method of strained parameters, a perturbation technique (Nayfeh, 1981). We chose the perturbation parameter as the amplitude of the speed fluctuations because the varying component of the spindle speed is small compared to the constant mean part. Analytical expressions for determining stability are presented in this section, and the relevant numerical results are given in the next section.

\subsection{Formulation of the Problem}

Following Tsao, McCarthy, and Kapoor (1993), we write the single-degree-of-freedom regenerative type chatter equation as follows:

$$
\omega^{2}(\theta) x^{\prime \prime}(\theta)+\left[2 \zeta+\omega^{\prime}(\theta)\right] \omega(\theta) x^{\prime}(\theta)+(1+K) x(\theta)=K x(\theta-2 \pi) .
$$

All variables are in angle coordinates $\theta$, and prime denotes differentiation with respect to this coordinate. This form is convenient, especially for our perturbation analysis, because the time varying delay term is converted to a constant delay term. Because $t=t(\theta)$ is a monotonic function, the stability analysis in the $\theta$ domain is similar to that in the $t$ domain (Chen, Ulsoy, and Koren, 1992). The variable $x(\theta)$ is the relative cutter-workpiece displacement, $\omega$ is the spindle speed, $\zeta$ is the damping, and $K$ is the cutting force coefficient. All quantities in equation (1) are nondimensional. They are obtained from the dimensional quantities (denoted by asterisks) through the following relations:

$$
x=\frac{x^{*}}{L}, \quad \omega=\frac{\omega^{*}}{\omega_{n}}, \quad K=\frac{K^{*}}{\omega_{n}^{2}},
$$

where $L$ is a reference length and $\omega_{n}$ is the fundamental natural frequency of the system.

To proceed further, we assume that the spindle speed variation is of the following form:

$$
\omega=\omega_{0}(1+\varepsilon \sin \Omega \theta),
$$


where $\omega_{0}$ is the constant mean spindle speed and $\varepsilon$ and $\Omega$ are the amplitude and frequency of the speed variation, respectively. In practical applications, the fluctuations are small compared to the mean velocity, and hence $\varepsilon<<1$. This justifies taking $\varepsilon$ as the perturbation parameter in search of approximate solutions.

\subsection{Approximate Analytical Solution}

Before applying perturbations, we first transform equation (1) by defining the new variable $y(\theta)$ as follows:

$$
x(\theta)=e^{\gamma \theta} y(\theta) .
$$

This form is more convenient in representing the growing and decaying solutions observed in chatter vibrations. Substituting equation (4) into equation (1), we have

$$
\omega^{2} y^{\prime \prime}+\left[2 \gamma \omega^{2}+\left(2 \zeta+\omega^{\prime}\right) \omega\right] y^{\prime}+\left[\omega^{2} \gamma^{2}+\left(2 \zeta+\omega^{\prime}\right) \omega \gamma+1+K\right] y=K e^{-2 \pi y} y(\theta-2 \pi) \text {. }
$$

We seek solutions in terms of our natural perturbation parameter $\varepsilon$ as follows:

$$
y=y_{0}+\varepsilon y_{1}+\varepsilon^{2} y_{2}+\cdots
$$

We find that the method of strained parameters (Nayfeh, 1981) is best suited as a perturbation technique for this specific problem. First, $\gamma$ is expanded as follows:

$$
\gamma=\gamma_{0}\left(1+\varepsilon \gamma_{1}+\varepsilon^{2} \gamma_{2}+\cdots\right)
$$

Substituting equations (3), (6), and (7) into equation (5) and separating terms at each order of $\varepsilon$, we obtain

Order 1

$$
\omega_{0}^{2} y_{0}^{\prime \prime}+\left[2 \gamma_{0} \omega_{0}^{2}+2 \zeta \omega_{0}\right] y_{0}^{\prime}+\left[\omega_{0}^{2} \gamma_{0}^{2}+2 \zeta \omega_{0} \gamma_{0}+1+K\right] y_{0}-K e^{-2 \pi \gamma_{0}} y_{0}(\theta-2 \pi)=0 \text {. }
$$

\section{Order $\varepsilon$}

$$
\begin{aligned}
& \omega_{0}^{2} y_{1}^{\prime \prime}+\left[2 \gamma_{0} \omega_{0}^{2}+2 \zeta \omega_{0}\right] y_{1}^{\prime}+\left[\omega_{0}^{2} \gamma_{0}^{2}+2 \zeta \omega_{0} \gamma_{0}+1+K\right] y_{1}-K e^{-2 \pi \gamma_{0}} y_{1}(\theta-2 \pi)= \\
& -2 \omega_{0}^{2} \sin \Omega \theta y_{0}^{\prime \prime}-\left[2 \gamma_{0} \omega_{0}^{2}\left(\gamma_{1}+2 \sin \Omega \theta\right)+\omega_{0}\left(2 \zeta \sin \Omega \theta+\omega_{0} \Omega \cos \Omega \theta\right)\right] y_{0}^{\prime} \\
& -\left[\omega_{0}^{2} \gamma_{0}^{2}\left(2 \gamma_{1}+2 \sin \Omega \theta\right)+\gamma_{0} \omega_{0}\left(2 \zeta \gamma_{1}+2 \zeta \sin \Omega \theta+\omega_{0} \Omega \cos \Omega \theta\right)\right] y_{0} \\
& -2 \pi \gamma_{0} \gamma_{1} K e^{-2 \pi \gamma_{0}} y_{0}(\theta-2 \pi) .
\end{aligned}
$$

Order $\varepsilon^{2}$

$$
\begin{aligned}
& \omega_{0}^{2} y_{2}^{\prime \prime}+\left[2 \gamma_{0} \omega_{0}^{2}+2 \zeta \omega_{0}\right] y_{2}^{\prime}+\left[\omega_{0}^{2} \gamma_{0}^{2}+2 \zeta \omega_{0} \gamma_{0}+1+K\right] y_{2}-K e^{-2 \pi \gamma_{0}} y_{2}(\theta-2 \pi)= \\
& -2 \omega_{0}^{2} \sin \Omega \theta y_{1}^{\prime \prime}-\omega_{0}^{2} \sin ^{2} \Omega \theta y_{0}^{\prime \prime}-\left[2 \gamma_{0} \omega_{0}^{2}\left(\gamma_{1}+2 \sin \Omega \theta\right)+\omega_{0}(2 \zeta \sin \Omega \theta\right. \\
& \left.\left.+\omega_{0} \Omega \cos \Omega \theta\right)\right] y_{1}^{\prime}-\left[2 \gamma_{0} \omega_{0}^{2}\left(\gamma_{2}+2 \gamma_{1} \sin \Omega \theta+\sin ^{2} \Omega \theta\right)+\omega_{0}^{2} \Omega \sin \Omega \theta \cos \Omega \theta\right] y_{0}^{\prime} \\
& -\left[\omega_{0}^{2} \gamma_{0}^{2}\left(2 \gamma_{1}+2 \sin \Omega \theta\right)+\gamma_{0} \omega_{0}\left(2 \zeta \gamma_{1}+2 \zeta \sin \Omega \theta+\omega_{0} \Omega \cos \Omega \theta\right)\right] y_{1} \\
& -\left[\omega_{0}^{2} \gamma_{0}^{2}\left(\sin ^{2} \Omega \theta+4 \gamma_{1} \sin \Omega \theta+\gamma_{1}^{2}+2 \gamma_{2}\right)+\omega_{0} \gamma_{0}\left(2 \zeta \gamma_{2}+2 \zeta \gamma_{1} \sin \Omega \theta\right.\right. \\
& \left.\left.+\omega_{0} \Omega \cos \Omega \theta\left(\gamma_{1}+\sin \Omega \theta\right)\right)\right] y_{0}+K e^{-2 \pi \gamma_{0}}\left[-2 \pi \gamma_{0} \gamma_{1} y_{1}(\theta-2 \pi)-2 \pi \gamma_{0} \gamma_{2} y_{0}(\theta-2 \pi)\right. \\
& \left.+2 \pi^{2} \gamma_{0}^{2} \gamma_{1}^{2} y_{0}(\theta-2 \pi)\right] .
\end{aligned}
$$


At order 1, we assume a solution of the form

$$
y_{0}=A e^{i \frac{\eta}{\omega_{0}} \theta}+c c
$$

where $A$ is a complex constant and $c c$ stands for the complex conjugate of the preceding terms. Substituting equation (11) into (8), separating real and imaginary parts, we obtain

$$
\begin{gathered}
-\eta^{2}+\omega_{0}^{2} \gamma_{0}^{2}+2 \zeta \omega_{0} \gamma_{0}+1+K=K e^{-2 \pi \gamma_{0}} \cos \left(2 \pi \frac{\eta}{\omega_{0}}\right) \\
2\left(\omega_{0} \gamma_{0}+\zeta\right) \eta=-K e^{-2 \pi \gamma_{0}} \sin \left(2 \pi \frac{\eta}{\omega_{0}}\right) .
\end{gathered}
$$

For a given mean spindle speed and cutting force coefficient $K(\zeta$ is assumed fixed for a specific system), equations (12) and (13) can be considered as two nonlinear algebraic equations with unknowns $\gamma_{0}$ and $\eta$. Once these terms are solved, from equations (11) and (4), the constant spindle speed solutions are retrieved, the theory of which is well established. It turns out that there are an infinite number of $\left(\gamma_{0}, \eta\right)$ pairs satisfying equations (12) and (13). This fact is pointed out in Nayfeh, Chin, and Pratt (1996) also. If all $\gamma_{0}$ are negative, the solutions are decaying, and if at least one of the $\gamma_{0}$ values are positive, the solutions are exponentially growing. The $\gamma_{0}=0$ condition yields the well-known stability lobes separating the stable solutions from the unstable solutions. The stability lobes and the solutions of equations (12) and (13) will be further discussed in the Numerical Results section.

At order $\varepsilon$, we substitute $y_{0}$ given in (11) into the right-hand side of equation (9). Three different cases arise: (1) $\Omega \cong 0$, (2) $\Omega \cong 2 \eta / \omega_{0}$ and (3) $\Omega$ away from 0 or $2 \eta / \omega_{0}$. In the previous work, the frequency values for suppression of chatter are usually chosen to be not very close to zero, hence the $\Omega \cong 0$ case will be discarded. Case 2 yields very high fluctuation frequencies, which are hard to attain practically. In fact, this case is a resonant case and destabilizes the system rather than stabilizing it even at order $\varepsilon$. For bounded solutions of the constant spindle speed $\left(\gamma_{0}=0\right)$, it can be shown that the boundaries separating stable and unstable solutions in the frequency-amplitude $(\Omega-\varepsilon)$ plane are

$$
\Omega=\frac{2 \eta}{\omega_{0}} \pm \varepsilon \frac{\eta \zeta}{\omega_{0} \sqrt{\zeta^{2}+\eta^{2}}} .
$$

When damping is extremely small, this region tends to close, as can be verified from the second term. The frequency $\eta$ is usually an $O(1)$ term, and if the dimensionless speed $\omega_{0}=0.2$, then $\Omega \cong 10$, which is very high compared to the practically used frequency range of $0<\Omega<0.4$ (Tsao, McCarthy, and Kapoor, 1993). For systems with very high spindle speeds, $2 \eta / \omega_{0}$ values may decrease drastically, making it necessary to consider this resonant frequency. To the best of the authors' knowledge, this primary resonant frequency has not been reported.

To obtain the stabilizing mechanism reported theoretically and experimentally in previous studies, we therefore consider that the fluctuating frequency is always away from 0 or $2 \eta / \omega_{0}$ (i.e., Case 3 ). Elimination of secular terms for this case yields

$$
\gamma_{1}=0
$$


A solution for $y_{1}$ can therefore be written in the form

$$
y_{1}=c_{1} A e^{i\left(\Omega+\frac{\eta}{\omega_{0}}\right) \theta}+c_{2} \bar{A} e^{i\left(\Omega-\frac{\eta}{\omega_{4}}\right) \theta}+c c,
$$

where $c_{1}$ and $c_{2}$ are complex constants defined in the appendix (equations (A1) through (A10)).

At order $\varepsilon^{2}$, we substitute equations (11) and (16) to the right-hand side of equation (10). Two different cases arise: (1) $\Omega \cong \eta / \omega_{0}$ and (2) $\Omega$ away from $\eta / \omega_{0}$. Again, $\eta / \omega_{0}$ is a relatively high frequency, which in practice would be hard to track in working mechanisms at reasonable mean speeds and hence is discarded. For Case 2, then, elimination of secular terms yields

$$
\begin{aligned}
\operatorname{Re}\left(\gamma_{0} \gamma_{2}\right) & =\frac{1}{h_{1}^{2}+h_{2}^{2}}\left\{\frac{1}{2} h_{1}\left(\eta^{2}-\omega_{0}^{2} \gamma_{0}^{2}\right)-h_{2} \gamma_{0} \omega_{0} \eta+\frac{1}{g_{1}^{2}+g_{2}^{2}}\left[\left(h_{1} e_{1}+h_{2} e_{2}\right) \cdot\left(b_{1} g_{1}+b_{2} g_{2}\right)\right.\right. \\
& \left.-\left(h_{1} e_{2}-h_{2} e_{1}\right) \cdot\left(b_{2} g_{1}-b_{1} g_{2}\right)\right]+\frac{1}{g_{3}^{2}+g_{4}^{2}}\left[\left(h_{1} e_{3}+h_{2} e_{4}\right) \cdot\left(b_{3} g_{3}+b_{4} g_{4}\right)\right. \\
& \left.\left.+\left(h_{1} e_{4}-h_{2} e_{3}\right) \cdot\left(b_{4} g_{3}-b_{3} g_{4}\right)\right]\right\}
\end{aligned}
$$

where $h, g, b$, and $e$ terms are all defined in the appendix. We calculated only the real part of $\gamma_{0} \gamma_{2}$, since this is the component contributing to the stability of the system. Substituting equation (17) into (7), we have

$$
\gamma_{m}=\gamma_{0}+\varepsilon^{2} \operatorname{Re}\left(\gamma_{0} \gamma_{2}\right),
$$

where $\gamma_{m}$ is the approximate value of $\gamma$. The new stability boundaries can now be found by taking $\gamma_{m}=0$. Because we have an infinite number of $\gamma_{0}$, on the new stability boundary, only one of the $\gamma_{m}$ will be zero, with all others being negative numbers. Numerical calculations of $\gamma_{m}$, as well as the stability boundaries, will be presented in the next section.

Rather than numerically integrating the original equations, which requires high costs in terms of computational time, we presented an approximate analytical solution that requires solution of a nonlinear algebraic equation and evaluation of some coefficients, thereby reducing the computational time drastically. The analytical approach provides a better understanding of the dynamics of the problem. We obtain the resonant frequencies, which are hard to detect using pure numerical techniques. If the analysis had been carried out to higher orders, we would have found that the frequencies at which resonance may occur would be

$$
\Omega \cong \frac{2 \eta}{n \omega_{0}}, \quad n=1,2,3, \cdots
$$

For an increasing $n$, the resonances will be weaker, with the primary resonance corresponding to $n=1$. We showed that $n=1$ in fact does yield instability, but a further investigation of stability is needed for $n>1$. 


\section{NUMERICAL RESULTS}

Numerical results for constant spindle speed and the effect of speed variation on the stability of the system will be presented in this section.

\subsection{Constant Spindle Speed}

To determine the stability lobes analytically for constant speeds, we take $\gamma_{0}=0$ in equations (12) and (13):

$$
\begin{gathered}
-\eta^{2}+1+K=K \cos \left(2 \pi \frac{\eta}{\omega_{0}}\right) \\
2 \zeta \eta=-K \sin \left(2 \pi \frac{\eta}{\omega_{0}}\right) .
\end{gathered}
$$

Squaring and adding both equations, and solving for the frequency $\eta$, we have

$$
\eta=\sqrt{1+K-2 \zeta^{2} \pm \sqrt{(K+2 \zeta(1-\zeta))(K-2 \zeta(1+\zeta))}}
$$

For a given $\zeta$ and $K$, the two different $\eta$ values correspond to the left and right arms of a stability lobe. For the case of

$$
K=2 \zeta(\zeta+1)
$$

we obtain the minimum points of all stability lobes, that is, the line represents the tangent line to the minimum points of all stability lobes. Below this value of $K$, we always have stability irrespective of the mean velocity.

Substituting equation (22) into (20) and solving for $\omega_{0}$, we have

$$
\omega_{0}=\frac{2 \pi \sqrt{1+K-2 \zeta^{2} \pm \sqrt{(K+2 \zeta(1-\zeta))(K-2 \zeta(1+\zeta))}}}{2 \pi-\operatorname{Arccos}\left\{\frac{2 \zeta^{2} \pm \sqrt{(K+2 \zeta(1-\zeta))(K-2 \zeta(1+\zeta))}}{K}\right\}+2 n \pi}, \quad n=0,1,2,3, \ldots
$$

Each $n$ corresponds to a different lobe. In Figure 1, the stability lobes for $\zeta=0.005$ (solid) and the stability lobes for $\zeta=0.05$ (dashed) are presented. There is a net stability gain when damping is larger, as can be seen also from equation (23). Figure 1 (solid lines) is similar to that given in Tsao, McCarthy, and Kapoor (1993, Figure 4), with improvements in our results at lower spindle speeds. Note also that the spindle speed range considered is greater in our figure.

The stable (decaying) solutions under the stability lobes and the unstable (growing) solutions above the stability lobes can be detected analytically by solving equations (12) and (13) for a given $\omega_{0}$ and $K$ value. Although the equation modeling the motion is a second-order differential-difference equation, due to the delay term, the modes of vibrations are infinite in agreement with Nayfeh, Chin, and Pratt (1996). We therefore have an infinite number of $\left(\gamma_{0}, \eta\right)$ pairs. Below the stability lobes, all $\gamma_{0}$ are negative, and hence the solutions are decaying. Exactly on the stability lobes, one $\gamma_{0}$ is zero while all others 


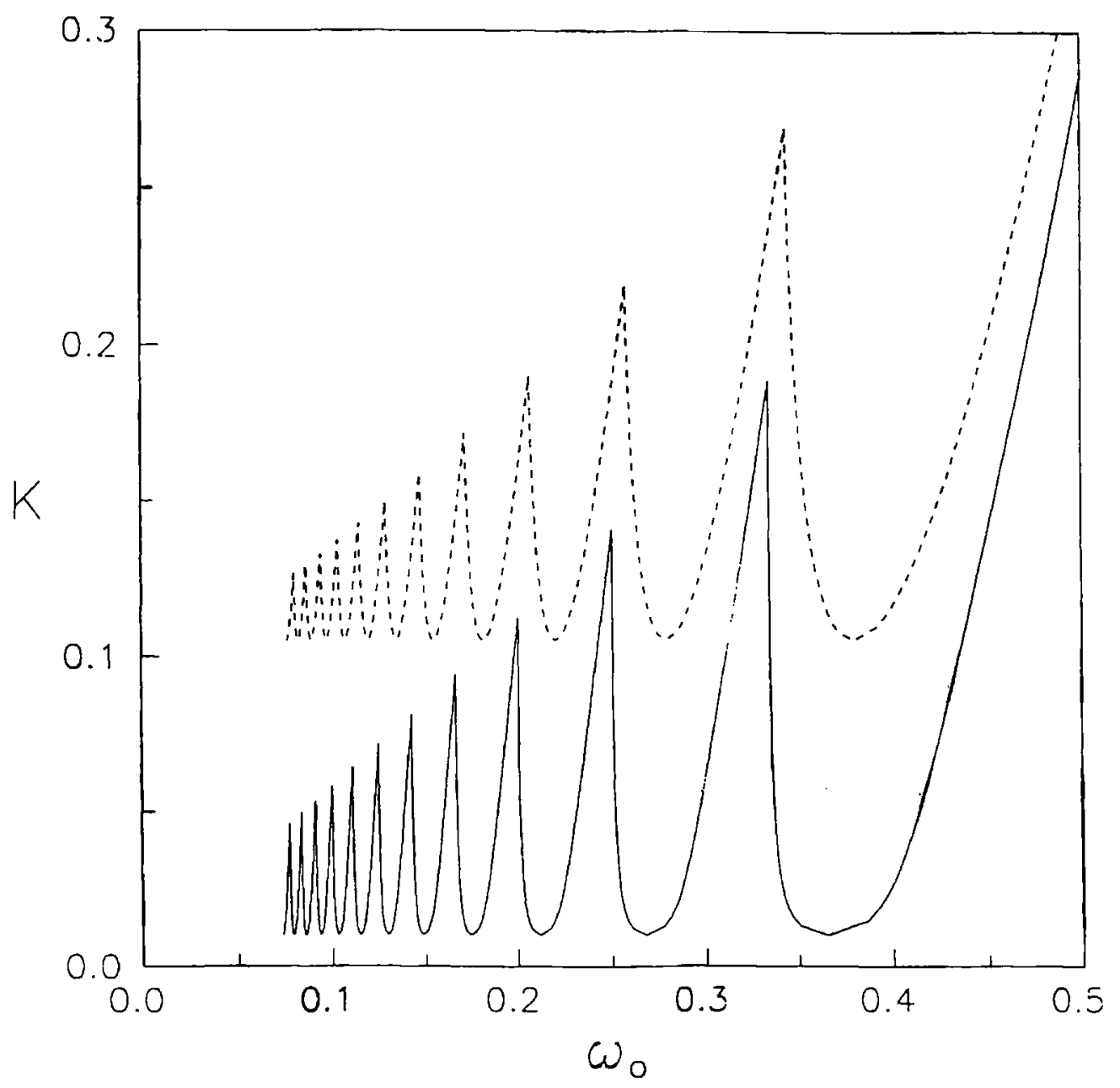

Figure 1. Stability lobes for constant spindle speed case with damping $\zeta=0.005$ (solid) and $\zeta=0.05$ (dashed).

are negative (decaying in time). Above the stability lobes, one or more $\gamma_{0}$ are positive, and the solutions grow in time. Numerical values of some of the $\left(\gamma_{0}, \eta\right)$ pairs are presented in Table 1 and in Table 2 for sample growing solutions. Note that only the largest several roots are presented.

We also integrate numerically the original differential-difference equation using a Runge-Kutta method. In the first revolution $(0<\theta<2 \pi)$, the delay term is taken as zero, and in the following revolutions, the delay term is taken into account as in Nayfeh, Chin, and Pratt (1996). Sample plots are given for stable (Figure 2), bounded (Figure 3), and unstable (Figure 4) solutions.

\subsection{Spindle Speed Variation}

When we superpose the harmonic fluctuations onto the constant mean spindle speed, our perturbation solution indicates that there is a correction term of $\varepsilon^{2} \operatorname{Re}\left(\gamma_{0} \gamma_{2}\right)$ to the 
Table 1. Growing factor $\left(\gamma_{0}\right)$ and frequency $(\eta)$ of the constant speed solution and the corresponding growing factor $\left(\gamma_{m}\right)$ of the variable speed solution $\left(\omega_{0}=0.112, K=0.045, \zeta=\right.$ $0.005, \varepsilon=0.02, \Omega=0.4$ ).

\begin{tabular}{ccc}
\hline$\gamma_{0}$ & $\eta$ & $\gamma_{m}$ \\
\hline-0.681135 & 2.070379 & -0.681066 \\
-0.657317 & 1.958285 & -0.657241 \\
-0.630988 & 1.846174 & -0.630902 \\
-0.601461 & 1.734039 & -0.601361 \\
-0.567702 & 1.621869 & -0.567580 \\
-0.528038 & 1.509647 & -0.527881 \\
-0.499036 & 0.112197 & -0.499035 \\
-0.493139 & 0.224403 & -0.493138 \\
-0.482798 & 0.336630 & -0.482793 \\
-0.479484 & 1.397340 & -0.479260 \\
-0.467083 & 0.448895 & -0.467072 \\
-0.444278 & 0.561221 & -0.444253 \\
-0.415818 & 1.284881 & -0.415437 \\
-0.411033 & 0.673658 & -0.410973 \\
-0.359801 & 0.786299 & -0.359625 \\
-0.319897 & 1.172134 & -0.318925 \\
-0.267081 & 0.899334 & -0.266207 \\
-0.096174 & 1.061338 & -0.082837 \\
0.011508 & 1.002546 & -0.005055 \\
\hline
\end{tabular}

Table 2. Growing factor $\left(\gamma_{0}\right)$ and frequency $(\eta)$ of the constant speed solution and the corresponding growing factor $\left(\gamma_{m}\right)$ of the variable speed solution $\left(\omega_{0}=0.112, K=0.057\right.$, $\zeta=0.005, \varepsilon=0.02, \Omega=0.4$ ).

\begin{tabular}{cccc} 
& $\gamma_{0}$ & \multicolumn{1}{c}{$\boldsymbol{\eta}$} & $\gamma_{m}$ \\
\cline { 2 - 4 } & $\cdot$ & $\dot{\cdot}$ & $\dot{\cdot}$ \\
-0.684937 & 2.294607 & -0.684877 \\
-0.664764 & 2.182543 & -0.664700 \\
-0.642897 & 2.070470 & -0.642827 \\
& -0.618979 & 1.958386 & -0.618902 \\
& -0.592520 & 1.846285 & -0.592433 \\
& -0.562820 & 1.734164 & -0.562718 \\
-0.528816 & 1.622012 & -0.528692 \\
-0.488790 & 1.509816 & -0.488628 \\
-0.463187 & 0.112179 & -0.463187 \\
-0.457355 & 0.224366 & -0.457354 \\
-0.447133 & 0.336572 & -0.447128 \\
-0.439644 & 1.397551 & -0.439413 \\
-0.431614 & 0.448808 & -0.431603 \\
-0.409129 & 0.561096 & -0.409104 \\
-0.376441 & 0.673472 & -0.376382 \\
-0.374865 & 1.285168 & -0.374465 \\
-0.326336 & 0.786000 & -0.326166 \\
-0.276157 & 1.172626 & -0.275098 \\
-0.236997 & 0.898743 & -0.236198 \\
-0.044078 & 1.064032 & -0.036137 \\
0.014651 & 1.003379 & 0.003437 \\
\hline
\end{tabular}




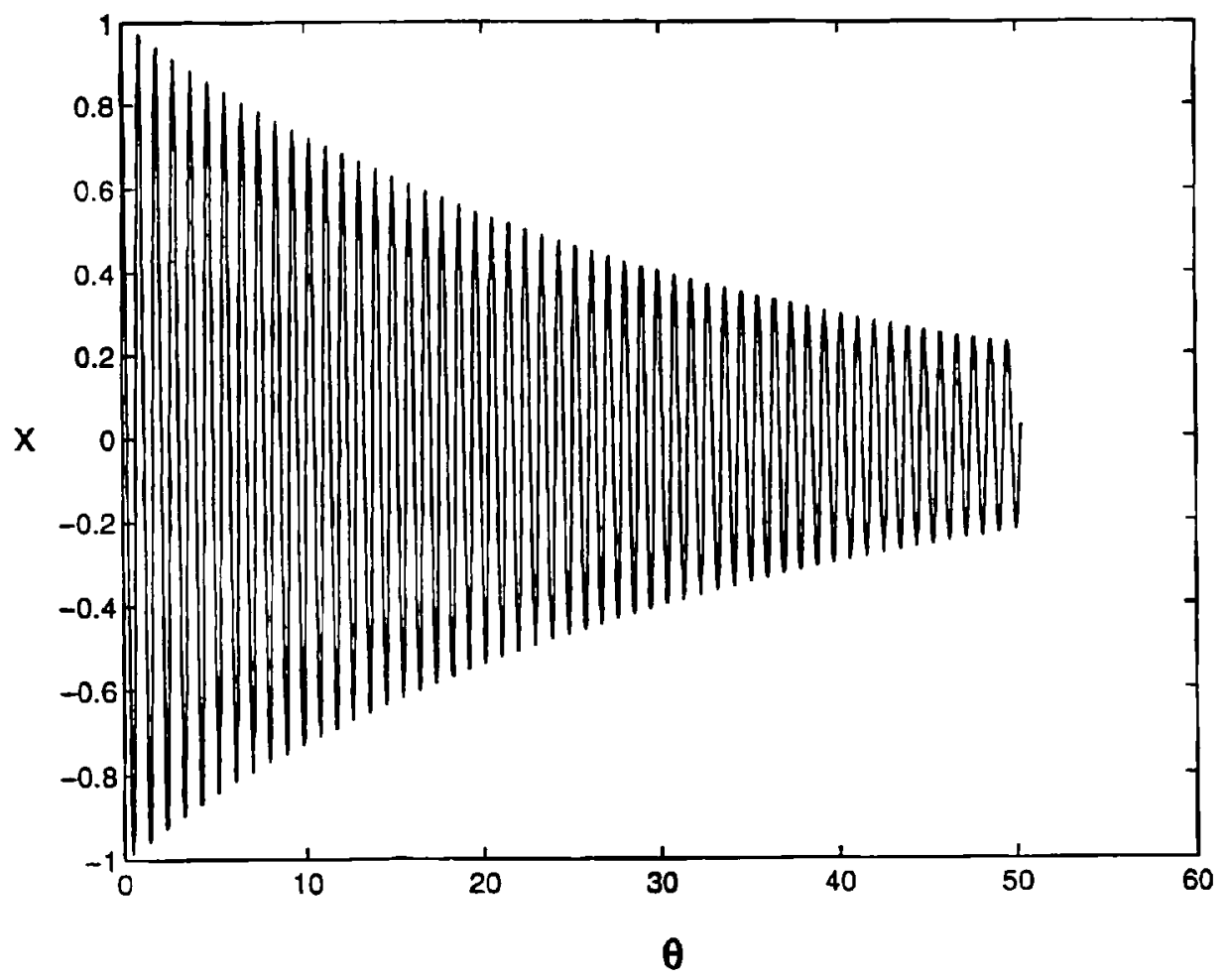

Figure 2. Numerical simulation for a constant speed stable solution $(\zeta=0.005, \zeta=0.14887, K=0.001)$.

original $\gamma_{0}$ term that determines the stability. Therefore, for each root $\gamma_{0}$, the correction term should be calculated and added to $\gamma_{0}$, thus altering the stability. These modified values $\left(\gamma_{m}\right)$ are also given in Table 1 and Table 2 for $\varepsilon=0.02$ and $\Omega=0.4$. Note that for $K=0.045$ (Table 1 ), the unstable constant speed solutions can be stabilized, whereas for $K=0.057$ (Table 2), solutions cannot be stabilized.

To determine the new stability lobes corresponding to variable spindle speed, we first choose a fixed $\omega_{0}$ value. We start from a low $K$ value and calculate the corresponding ( $\gamma_{0}$, $\eta)$ pairs from equations (12) and (13). The range $-1<\gamma_{0}<1$ is enough to consider for practical purposes. We then calculate the modified $\gamma_{m}$ values and check their sign. If all are negative, we increment $K$ and check the signs again. We repeat the procedure until at least one of the $\gamma_{m}$ is positive. The specific $K$ value then becomes an upper limit for stability. Repeating the same procedure for different $\omega_{0}$ values, we determine the new stability borders. For $\varepsilon=0.02$ and $\Omega=0.4$, the stability lobes for variable spindle speed (dashed lines) are compared to those of constant spindle speed (solid lines) in Figure 5. There is always gain in stability, although small, for an amplitude of speed fluctuation of $2 \%$. We also observed that gains are less for $\Omega=0.7$ compared to the case of $\Omega=0.4$. This shows the importance of selecting an optimum amplitude and frequency.

Up to $\varepsilon=0.02$, the stabilizing mechanism works by changing only the sign of the single positive $\gamma_{0}$ root. For greater values of $\varepsilon$, the stabilizing effect on the positive root increases. However, a change in sign of the next closest negative root occurs, which 


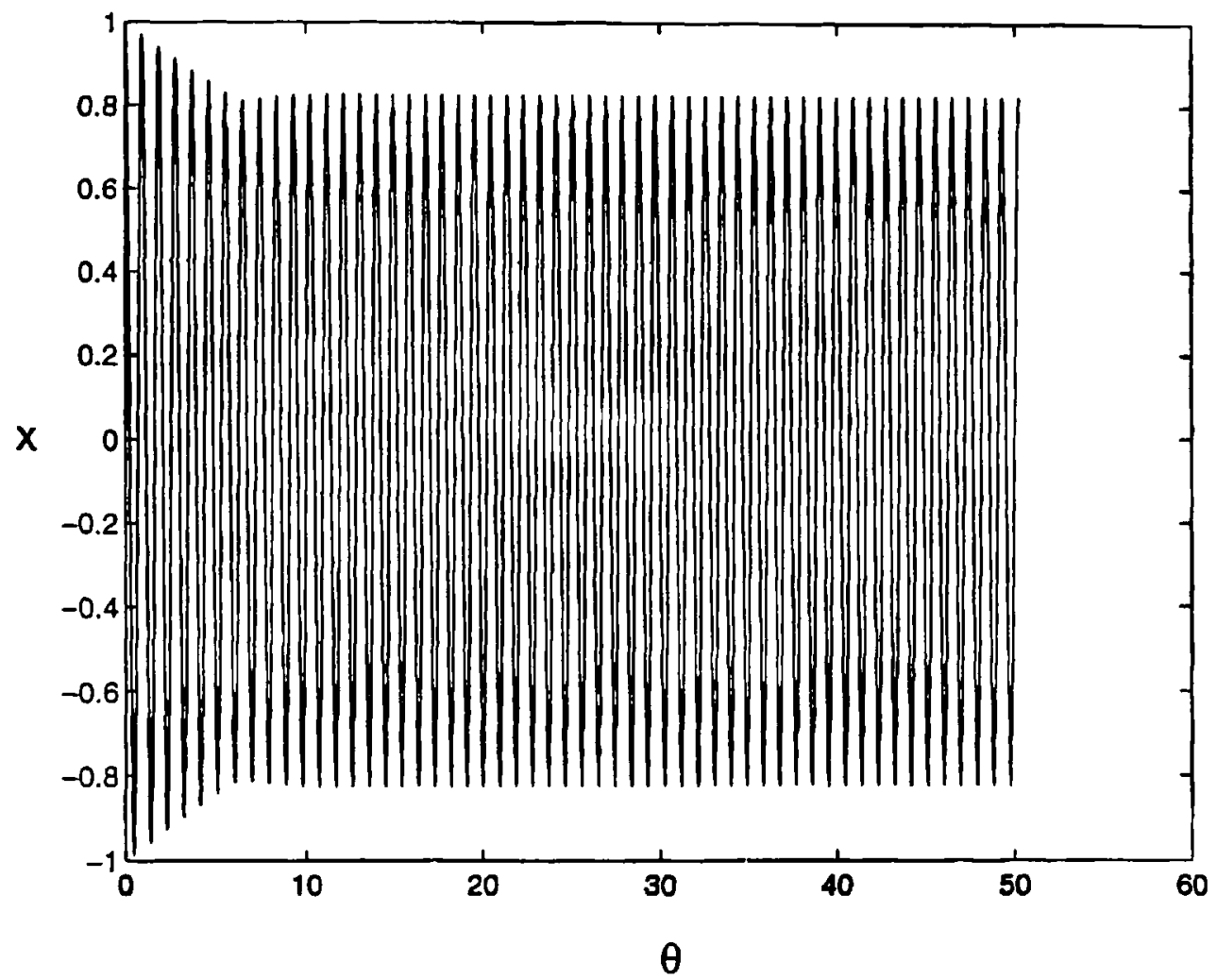

Figure 3. Numerical simulation for a constant speed bounded solution $(\zeta=0.005, \zeta=0.14887, K=0.01005)$.

destabilizes the system. This mechanism may explain the reason of the worse solutions for $\varepsilon=0.1$ compared to the constant speed solutions reported in Tsao McCarthy, and Kapoor (1993, Figure 6). However, we could not interpret our results for $\varepsilon>0.02$ because for some $\omega_{0}$ values, a single stability border could not be achieved. This may be due to the truncation of the perturbation series after three terms.

The stability borders for $\varepsilon=0.02$ are verified using numerical simulations. From Figure 5 or Table 1 , for $\omega_{0}=0.112$ and $K=0.045$, constant spindle speed solutions are unstable as also verified by numerical integrations of the original equation (Figure 6). When an amplitude of speed fluctuation of $2 \%$ and a frequency of 0.4 is implemented, the solution should be stable from Figure 5 or Table 1. This is verified numerically, as shown in Figure 7. $K=0.053$ represents the stability border theoretically for $\omega_{0}=0.112$ for the variable spindle speed case. Taking a slightly higher value of $K=0.057$, the solutions should be unstable (see Table 2 or Figure 5). This result is verified through numerical simulations, as shown in Figure 8. 


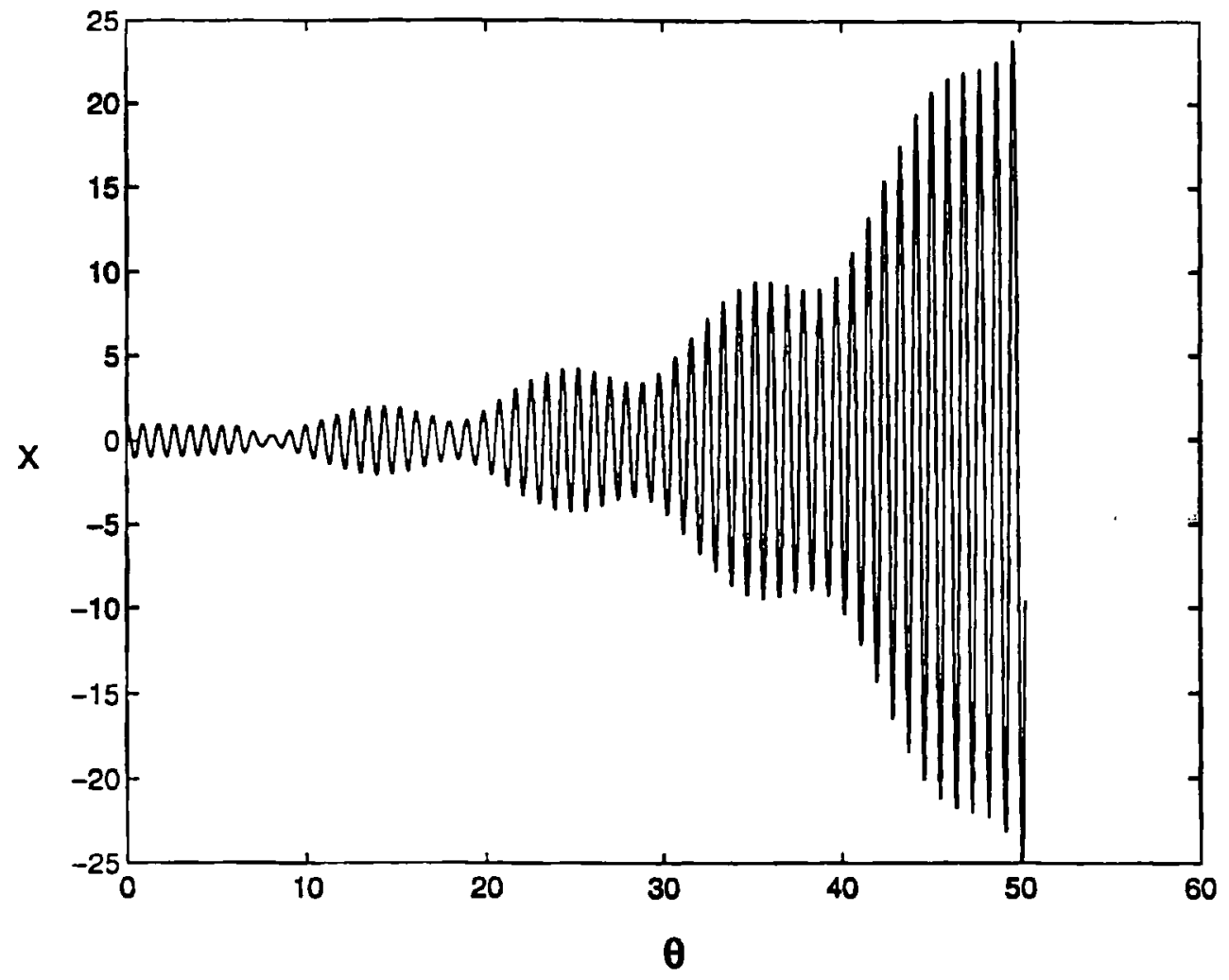

Figure 4. Numerical simulation for a constant speed unstable solution $(\zeta=0.005, \zeta=0.14887, K=0.15)$. 


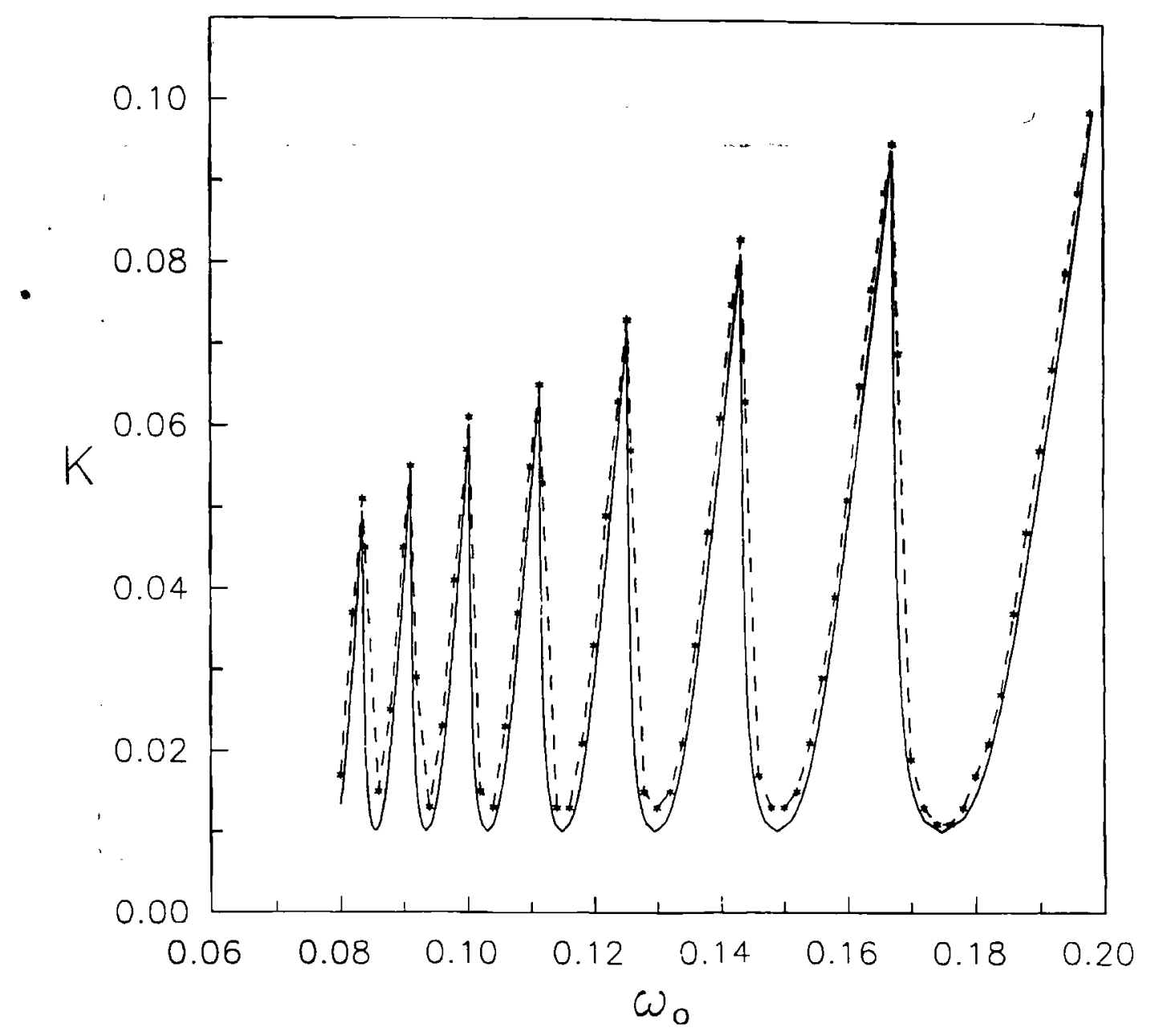

Figure 5. Comparison of stability lobes for constant spindle speeds (solid lines) and variable spindle speeds (dashed lines) $(\zeta=0.005, \varepsilon=0.02, \Omega=0.4)$. 


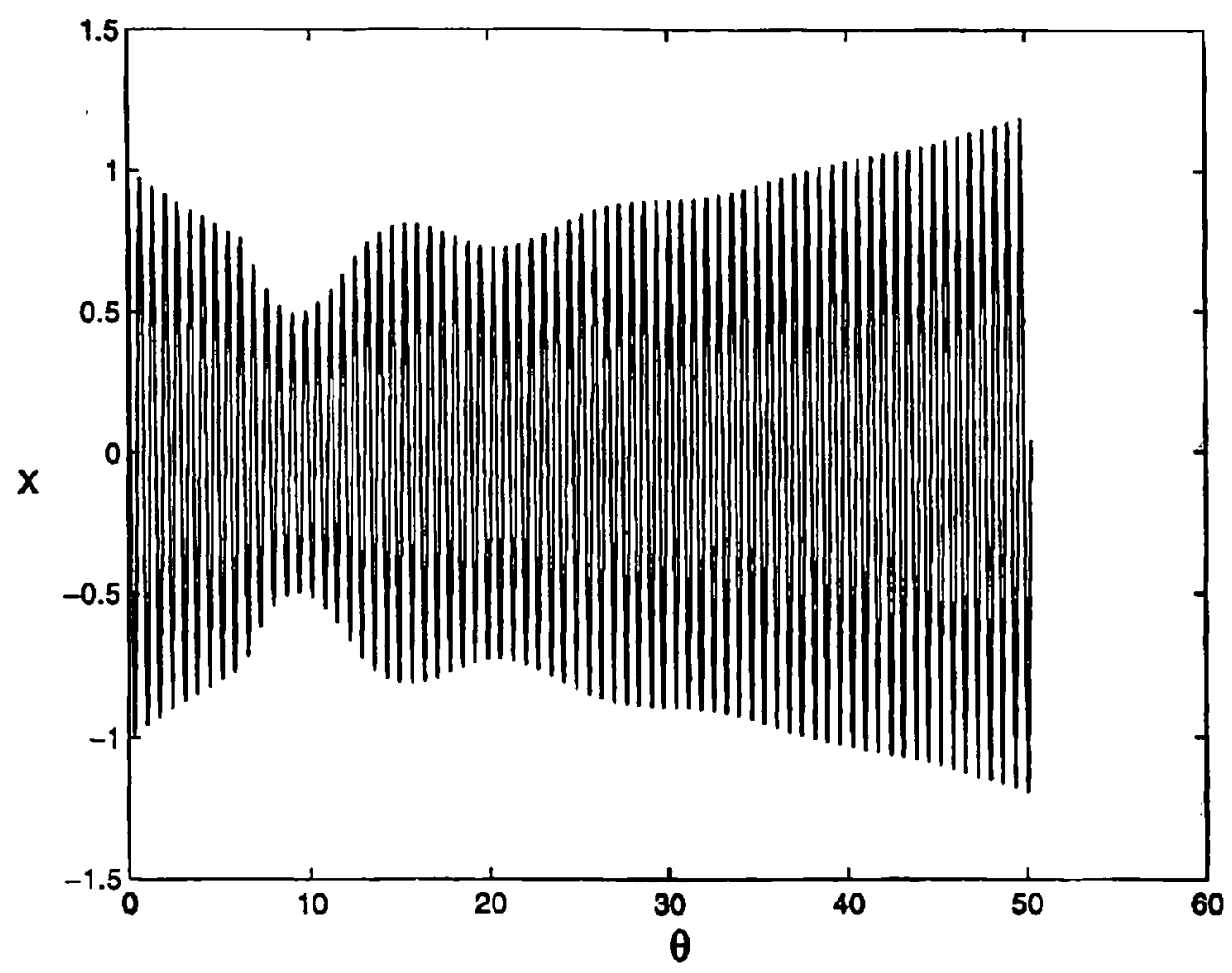

Figure 6. Numerical simulation for a constant speed solution $\left(\zeta=0.005, \omega_{0}=0.112, K=0.045\right)$. 


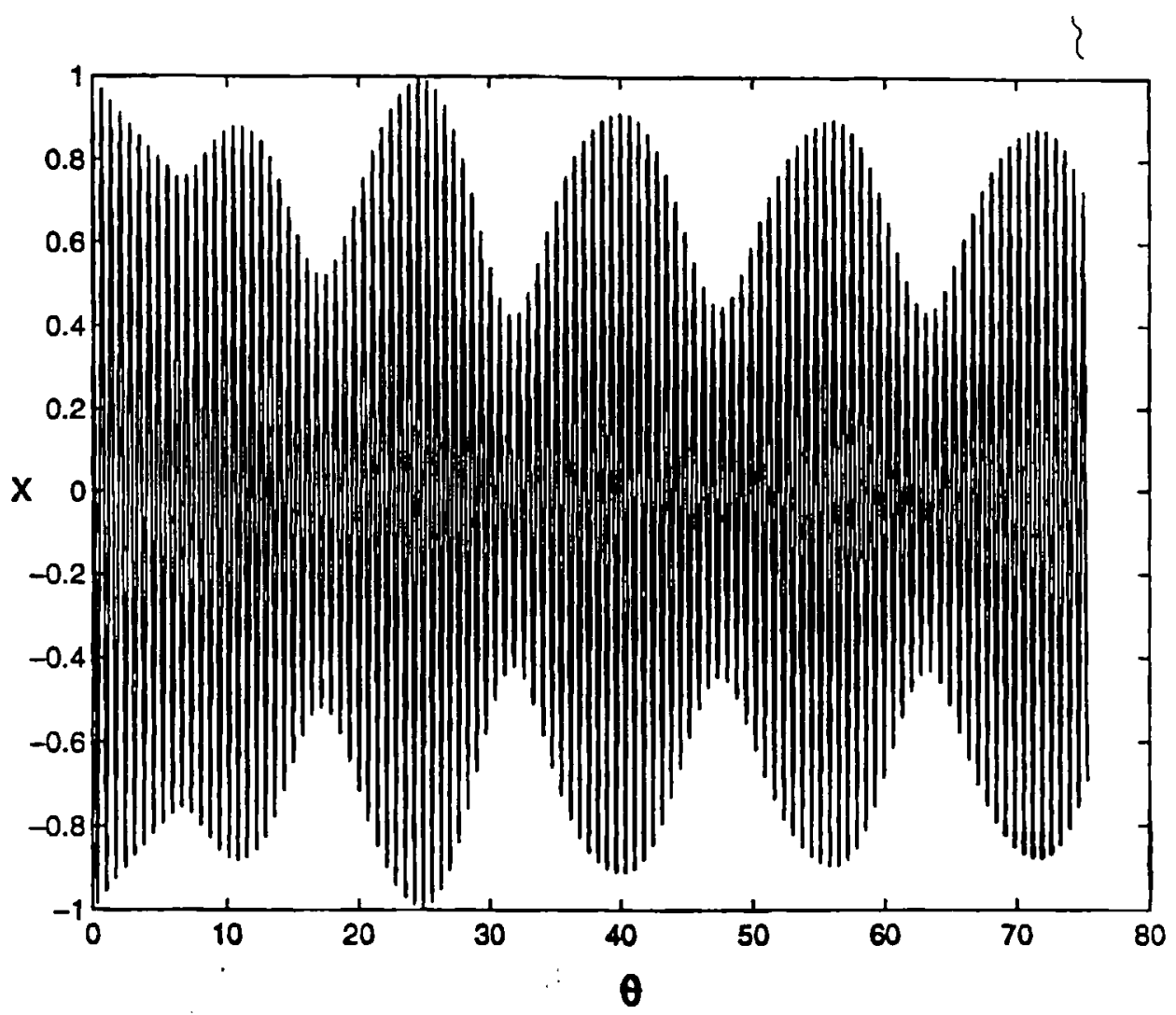

Figure 7. Numerical simulation for a variable speed stable solution $\left(\zeta=0.005, \omega_{0}=0.112, K=0.045, \varepsilon=0.02\right.$, $\Omega=0.4$. 


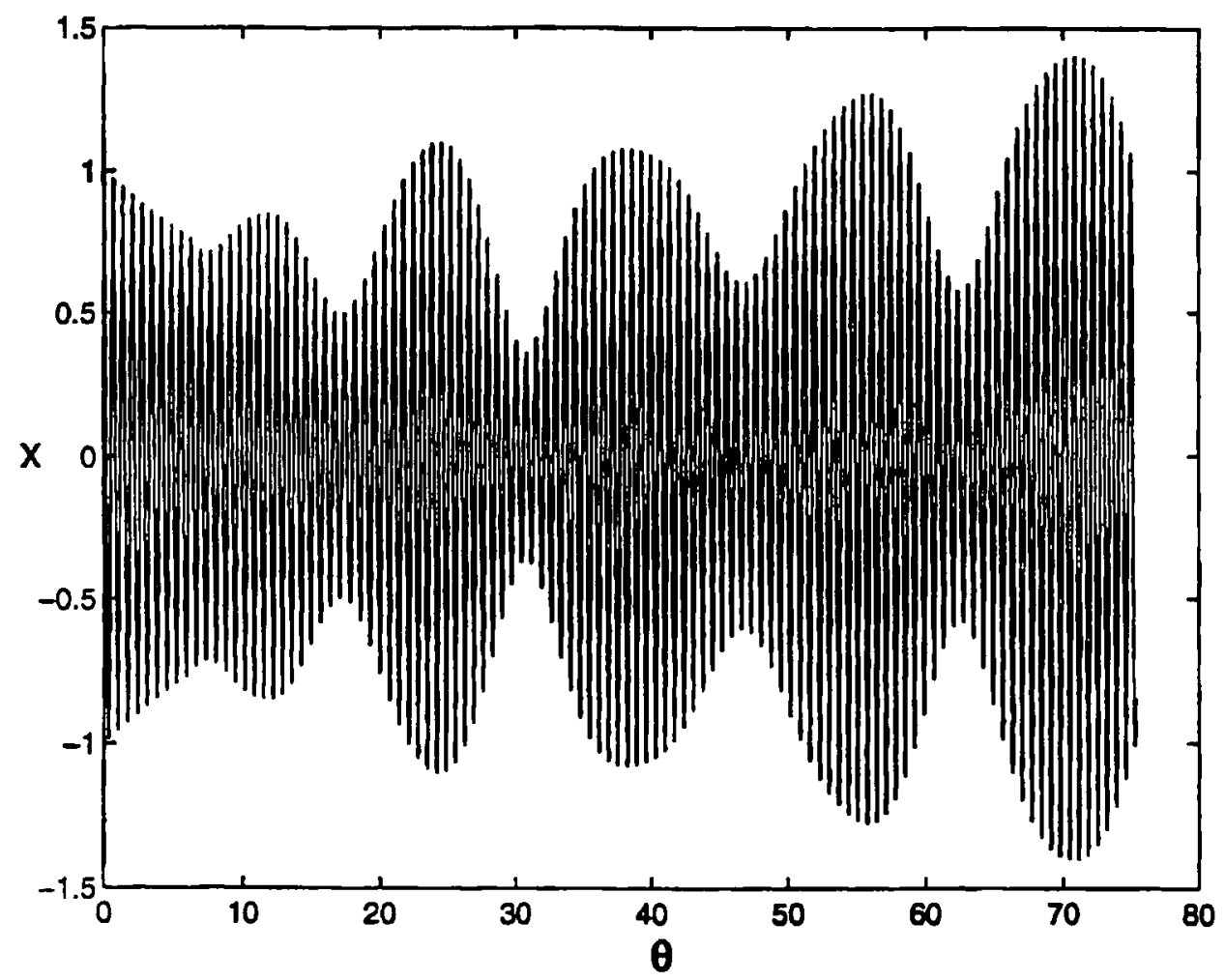

Figure 8. Numerical simulation for a variable speed unstable solution $\left(\zeta=0.005, \omega_{0}=0.112, K=0.057, \varepsilon=0.02\right.$, $\Omega=0.4)$. 


\section{CONCLUDING REMARKS}

Approximate analytical solutions using the method of strained parameters (a perturbation technique) are presented, for the first time, for a variable speed chatter problem. The resonant frequencies that may lead to instabilities are detected. Although the primary resonant frequencies are high, they can be reached in high-speed machining. The analysis gives better results for amplitudes of speed fluctuations not exceeding $2 \%$ of the mean spindle speed. For these values, although small, there is always an increase in the stability regions when the frequency is away from the first two resonances. To attain larger gains in stability, however, numerical results of previous work predicted required amplitudes of $20 \%$. These amplitudes are too high to get accurate results using our perturbation approach. Theoretical results, for small amplitudes, have been verified using numerical simulations of the original equation by the Runge-Kutta method.

This work can be improved through further research in a number of ways. The range of validity of the results may be increased by some transformation techniques and different expansions. The speed variation problem may also be investigated using a nonlinear version of the problem. The effectiveness of feed variation instead of speed variation can also be analyzed using perturbations.

\section{APPENDIX}

Definition of Complex Constants in Equations (16) and (17)

$$
\begin{aligned}
& c_{1}=\frac{b_{1}+i b_{2}}{g_{1}+i g_{2}} \\
& c_{2}=\frac{b_{3}+i b_{4}}{g_{3}+i g_{4}} \\
& b_{1}=-\eta\left(2 \gamma_{0} \omega_{0}+\zeta\right)-\frac{1}{2} \omega_{0}^{2} \Omega \gamma_{0} \\
& b_{2}=-\eta^{2}-\frac{1}{2} \omega_{0} \Omega \eta+\omega_{0} \gamma_{0}\left(\omega_{0} \gamma_{0}+\zeta\right) \\
& b_{3}=\eta\left(2 \gamma_{0} \omega_{0}+\zeta\right)-\frac{1}{2} \omega_{0}^{2} \Omega \gamma_{0} \\
& b_{4}=-\eta^{2}+\frac{1}{2} \omega_{0} \Omega \eta+\omega_{0} \gamma_{0}\left(\omega_{0} \gamma_{0}+\zeta\right) \\
& g_{1}=-\omega_{0}^{2}\left(\Omega+\frac{\eta}{\omega_{0}}\right)^{2}+\omega_{0}^{2} \gamma_{0}^{2}+2 \zeta \gamma_{0} \omega_{0}+1+K-K e^{-2 \pi \gamma_{0}} \cos \left\{2 \pi\left(\Omega+\frac{\eta}{\omega_{0}}\right)\right\} \\
& g_{2}=2 \omega_{0}\left(\gamma_{0} \omega_{0}+\zeta\right)\left(\Omega+\frac{\eta}{\omega_{0}}\right)+K e^{-2 \pi \gamma_{0}} \sin \left\{2 \pi\left(\Omega+\frac{\eta}{\omega_{0}}\right)\right\} \\
& g_{3}=-\omega_{0}^{2}\left(\Omega-\frac{\eta}{\omega_{0}}\right)^{2}+\omega_{0}^{2} \gamma_{0}^{2}+2 \zeta \gamma_{0} \omega_{0}+1+K-K e^{-2 \pi \gamma_{0}} \cos \left\{2 \pi\left(\Omega-\frac{\eta}{\omega_{0}}\right)\right\}
\end{aligned}
$$




$$
\begin{aligned}
& g_{4}=2 \omega_{0}\left(\gamma_{0} \omega_{0}+\zeta\right)\left(\Omega-\frac{\eta}{\omega_{0}}\right)+K e^{-2 \pi \gamma_{0}} \sin \left\{2 \pi\left(\Omega-\frac{\eta}{\omega_{0}}\right)\right\} \\
& h_{1}=2 \omega_{0}\left(\gamma_{0} \omega_{0}+\zeta\right)+2 \pi K e^{-2 \pi \gamma_{0}} \cos \left\{2 \pi \frac{\eta}{\omega_{0}}\right\} \\
& h_{2}=2 \omega_{0} \eta-2 \pi K e^{-2 \pi \gamma_{0}} \sin \left\{2 \pi \frac{\eta}{\omega_{0}}\right\} \\
& e_{1}=\omega_{0}\left(2 \gamma_{0} \omega_{0}+\zeta\right)\left(\Omega+\frac{\eta}{\omega_{0}}\right)-\frac{1}{2} \gamma_{0} \omega_{0}^{2} \Omega \\
& e_{2}=\omega_{0}^{2}\left(\Omega+\frac{\eta}{\omega_{0}}\right)^{2}-\frac{1}{2} \omega_{0}^{2} \Omega\left(\Omega+\frac{\eta}{\omega_{0}}\right)-\gamma_{0} \omega_{0}\left(\gamma_{0} \omega_{0}+\zeta\right) \\
& e_{3}=\omega_{0}\left(2 \gamma_{0} \omega_{0}+\zeta\right)\left(\Omega-\frac{\eta}{\omega_{0}}\right)-\frac{1}{2} \gamma_{0} \omega_{0}^{2} \Omega \\
& e_{4}=-\omega_{0}^{2}\left(\Omega-\frac{\eta}{\omega_{0}}\right)^{2}+\frac{1}{2} \omega_{0}^{2} \Omega\left(\Omega-\frac{\eta}{\omega_{0}}\right)+\gamma_{0} \omega_{0}\left(\gamma_{0} \omega_{0}+\zeta\right) .
\end{aligned}
$$

Acknowledgments. M. Pakdemirli acknowledges the support of the Scientific and Technical Research Council of Turkey (TUBITAK) in the form of a NATO scholarship during his stay at the University of Michigan.

\section{REFERENCES}

Amold, R. N., 1946, "The mechanism of tool vibration in the cutting of steel," Proceedings, Instit. of Mech. Eng. 154, 261. Chen, S. G., Ulsoy, A. G., and Koren, Y., 1992, "Modeling, simulation and analysis of regenerative chatter in tuming using the $\beta$ domain formulation," Proceedings of the IFAC Workshop on Intelligent Manufacturing Systems, Dearbom, MI, October.

Hanna, N. H. and Tobias, S. A., 1974, “A theory of nonlinear regenerative chatter," ASME J. Eng. Indust. 96, $247-255$.

Hoshi, et al., 1977, "Study for practical application of fluctuating speed cutting for regenerative chatter control," Annals of the CIRP 25, 175-179.

Inamura, T. and Sata, T., 1974, "Stability analysis of cutting under varying spindle speed," Annals of the CIRP 23, $119-120$. Inamura, T. and Sata, T., 1975, "Stability analysis of cutting under varying spindle speed," J. Fac. Eng. Tokyo Univ. 33, 1. Kondo, Y., Kawano, O., and Sato, H., 1981, "Behavior of self-excited chatter due to multiple regenerative effect," $A S M E$ J. Eng. Indust. 103, 324-329.

Lin, J. S. and Weng, C. I., 1990, "A nonlinear dynamic model of cutting," Int. J. Mach. Tools Manufact. 30, 53-64.

Lin, S. C., DeVor, R. E., and Kapoor, S. G., 1990, "The effects of variable speed cutting on vibration control in face milling," ASME J. Eng. Indust. 112, 1-11.

Nayfeh, A. H., 1981, Introduction to Perturbation Technigues, Wiley-Interscience, New York.

Nayfeh, A. H., Chin, C. M., and Pratt, J., 1997, "Perturbation methods in nonlinear dynamics-applications to machining dynamics," J. Manufact. Sci. Eng. 119.

Sexton, J. S., Milne, R. D., and Stone, B. J., 1977, "A stability analysis of single-point machining with varying spindle speed," Appl. Math. Modeling 1, 310-318.

Sexton, J. S. and Stone, B. J., 1978, "The stability of machining with continuously varying spindle speed," Annals of the CIRP 27, 321-326.

Sexton, J. S. and Stone, B. J., 1980, "An investigation of the transient effects during variable speed cutting," J. Mech. Eng. Sci. 22, 107-118.

Stoferle, T. and Grab, H., 1972, "Vermeiden von Ratterschwingungen durch Periodische Drehzahlanderung," Werkstatt und Betrieb 105, 727.

Takemura, T., Kitamura, T., and Hoshi, T., 1974, "Active suppression of chatter by programmed variation of spindle speed," Annals of the CIRP 23, 121-122.

Tobias, S. A., 1965, Machine Tool Vibration, John Wiley, New York.

Tsao, T. C., McCarthy, M. W., and Kapoor, S. G., 1993, "A new approach to stability analysis of variable speed machining systems," Int. J. Mach. Tools Manufact. 33, 791-808.

Welbourn, D. B. and Smith, J. D., 1970, Machine-Tool Dynamics: An Introduction, Cambridge University Press, Cambridge. 